\title{
Infantile hypertrophic pyloric stenosis: profile, management and outcome of patients admitted to a tertiary hospital in Bloemfontein, South Africa
}

\author{
R Arnold, ${ }^{1}$ K Khokho, ${ }^{1}$ K Narrainsamy, ${ }^{1}$ E Brits, ${ }^{2}$ G Joubert ${ }^{3}$ \\ ${ }^{1}$ Undergraduate medical student, Department of Surgery, Faculty of Health Sciences, University of the Free State, Bloemfon- \\ tein, South Africa \\ ${ }^{2}$ Department of Surgery, Faculty of Health Sciences, University of the Free State, Bloemfontein, South Africa \\ ${ }^{3}$ Department of Biostatistics, Faculty of Health Sciences, University of the Free State, Bloemfontein, South Africa
}

Corresponding author: Dr E Brits (BritsE@ufs.ac.za)

\begin{abstract}
Background: Infantile hypertrophic pyloric stenosis (IHPS) is the thickening of both muscle layers of the pylorus and is most commonly found in first-born males. It usually presents with projectile, non-bilious vomiting. Late presentation leads to severe dehydration and malnutrition with deranged serum-electrolytes and acid-base imbalance delaying treatment and prolonging hospital stay. This study aims to evaluate the profile, management and outcome of IHPS at a tertiary hospital in Bloemfontein, South Africa.

Methods: The study was a retrospective, descriptive record review including all patients with IHPS admitted to Universitas Hospital from January 2008 to February 2016. Of the 22 patients admitted, files for 19 patients were available for inclusion. Results: Sixteen $(84.2 \%)$ of the 19 patients were male. Of the 11 patients with available birth order, two were first-, two second-, six third- and one fourth-born. The patients' ages ranged from 27 to 194 days (median 51 days). The most common symptoms were projectile vomiting (78.9\%) and poor weight gain (68.4\%). Six patients had no ultrasound done, and 17 patients underwent a Ramstedt-pyloromyotomy. Eight patients received atropine as part of their initial management. The duration of symptoms ranged from 1 to 58 days (median 14 days). There was no reported mortality. The length of stay ranged from 2 to 60 days (median 7 days).

Conclusion: The gender distribution and age at presentation were in keeping with the literature but not the birth order. The delay before surgery emphasises the poor general health and deranged biochemical state the patients present at the hospital.
\end{abstract}

S Afr J Surg 2019;57(2)

http://dx.doi.org/10.17159/2078-5151/2019/v57n2a2811

\section{Introduction}

Infantile hypertrophic pyloric stenosis (IHPS) is the result of the thickening of both muscle layers of the pylorus and is characterised by projectile, non-bilious vomiting. It can progress to a near-complete obstruction of the gastric outlet, preventing chyme from entering the duodenum. ${ }^{1}$ This condition is most commonly found in first-born males with a reported ratio of males to females of $4: 1 .^{2}$ The incidence of IHPS has been reported to range from 0.5 to 5 per 1000 live births and is more common in Western countries ${ }^{3}$ than in African countries. ${ }^{4}$ Trends in different European regions are conflicting as to whether the disease is increasing or decreasing. 5
The incidence rate in Africa differs from country to country. A two-year retrospective analysis done at a teaching referral hospital in Ethiopia found that 61 out of 4729 infants admitted to the hospital were diagnosed with IHPS; a rate of 12.9 per 1000 admissions. ${ }^{6}$ In South Africa, a similar review was conducted over eight years from 2002 to 2010. Only 63 cases were recorded, which calculates to 0.18 per 1000 admissions. $^{?}$

Ultrasound is used to diagnose IHPS. ${ }^{8}$ Previously, the palpation of an olive-like mass in the epigastrium ${ }^{9}$ was regarded as pathognomonic, but the low-cost, highly accurate and non-invasive nature of ultrasound has become the gold standard in the diagnostic work-up. Infantile hypertrophic pyloric stenosis is generally managed surgically. Non-surgical 
methods may be indicated if surgery is not feasible, for instance when the baby's nutritional status does not allow for surgical intervention. ${ }^{7}$ The procedure most commonly used is a Ramstedt-pyloromyotomy, which can be done in either a minimally access or open manner. ${ }^{10}$ This procedure has $<0.5 \%$ mortality and $<10 \%$ morbidity rate. ${ }^{2}$ In developed countries, the mortality rate of IHPS has been reduced from $50 \%$ to almost $0 \%$, possibly because of advances in technology over the last few decades. ${ }^{11}$

Since IHPS is considered a rare condition in developing countries, more common conditions such as gastroenteritis, gastroesophageal reflux disease (GERD) and milk allergies are diagnosed when a patient presents with similar symptoms. As a result, IHPS is often overlooked and only considered after vomiting persists beyond therapeutic measures for the above-mentioned conditions. This contributes to the late presentation of infants to a paediatric surgical unit. Late presentation can lead to severe dehydration and malnutrition with deranged serum-electrolytes and acid-base imbalance. These imbalances may delay surgical intervention for several hours or days and may lead to increased length of hospital stay, morbidity and even mortality. Late presentation is the leading reason for complications, followed by misdiagnosis. ${ }^{2}$

Limited information is available on IHPS in developing countries such as South Africa. The fact that this is a rare condition in African populations contributes to this situation. Therefore, more information should be obtained on IHPS, including the role played by late presentations, misdiagnosis and other unique challenges in the outcome of these patients.

\section{Aim}

To determine the profile, management and outcome of patients admitted with infantile hypertrophic pyloric stenosis to a tertiary hospital in Bloemfontein.

\section{Materials and methods}

This study was a retrospective, descriptive record review. All patients with IHPS admitted to Universitas Hospital from January 2008 to February 2016 were included. It was expected that 20 to 25 patients would have been treated during this period.

\section{Measurement}

The Morbidity and Mortality $(\mathrm{M}+\mathrm{M})$ records of the Department of Surgery were scrutinised for the names and hospital numbers of all the patients treated with IHPS during the study period. The names and numbers were cross-referenced on the hospital electronic record system (Meditech). Patient files were collected from the hospital's records department. Relevant information from the files were captured by the student researchers on a data sheet and included demographic data, clinical signs and symptoms, laboratory results and radiological findings at admission. Data on the management of the patient included whether a Ramstedt-pyloromyotomy was performed and, if so, the date and time of procedure, use of atropine, complications during and after the surgery, postoperative outcomes, mortality and date of discharge.

\section{Pilot study}

A pilot study was conducted using the information of the first two patients. This did not result in any changes to the data sheet, and the two patient files were included in the main study.

\section{Analysis of data}

The data were entered into an Excel table. Data were analysed by the Department of Biostatistics, Faculty of Health Sciences, University of the Free State (UFS). The results were summarised by frequency and percentages (categorical variables) and percentiles (numerical variables).

\section{Ethical aspects}

Approval was obtained from the Health Sciences Research Ethics Committee of the University of the Free State (HSREC-S 09/2016) and the Free State Department of Health. No names, hospital numbers, or any other information that could lead to the identification of patients was captured on the data sheets in order to ensure patient confidentiality.

\section{Results}

Twenty-two patients were identified from the $\mathrm{M}+\mathrm{M}$ records. Three patient files could not be retrieved from the records department, and 19 patient files were included in the study. Sixteen $(84.2 \%)$ of these 19 patients were male. Of the 11 patients where the birth order was recorded, two were firstborn, two second-born, six third-born and one was fourthborn. Twelve patients (63.2\%) were younger than 60 days, while one $(5.3 \%)$ was older than 120 days (Table 1$)$. The patients' ages ranged from 27 to 194 days (median 51 days).

Table 1. Age, feeding and duration of symptoms

\begin{tabular}{lc}
\hline Variable & $\mathbf{n}(\mathbf{\%})$ \\
\hline Age at admission (days) $(\mathbf{n}=\mathbf{1 9})$ & $4(21.1)$ \\
$0-30$ & $8(42.1)$ \\
$31-60$ & $4(21.1)$ \\
$61-90$ & $2(10.5)$ \\
$91-120$ & $1(5.3)$ \\
$>120$ & \\
Types of feed $(\mathbf{n}=\mathbf{1 8})$ & $9(50.0)$ \\
Breastfeeding & $5(27.8)$ \\
Formula & $4(22.2)$ \\
Mixed feeding & \\
Duration of symptoms $($ days $)(\mathbf{n}=\mathbf{1 6})$ & $7(43.8)$ \\
$1-10$ & $6(37.5)$ \\
$11-20$ & $0(0)$ \\
$21-30$ & $3(18.8)$ \\
$>30$ & \\
&
\end{tabular}


Nine patients $(50.0 \%)$ were exclusively breastfed. The duration of symptoms before admission ranged from 1 to 58 days (median 14 days). Five patients $(31.3 \%)$ had a duration of symptoms for longer than 14 days.

Ultrasound was performed on 13 patients (68.4\%). Other conditions that were managed before the referral were noted for six patients $(31.6 \%)$ and included sepsis, milk allergy, gastroenteritis, aseptic meningitis and metabolic alkalosis.

The most common symptoms reported were projectile vomiting $(\mathrm{n}=15,78.9 \%)$ and poor weight gain $(\mathrm{n}=13$, $68.4 \%$ ) (Table 2). Voracious feeding, bloodstained vomiting, diarrhoea, non-projective vomiting or bilious vomiting were not reported for any of the patients.

Laboratory results showed that 15 patients $(78.9 \%)$ had a high $\mathrm{pH}(>7.45)$ and $11(57.9 \%)$ had low $\mathrm{PaCO}_{2}$ levels $(<33 \mathrm{mmHg})$. One patient $(5.3 \%)$ had raised infection markers (Table 2).

Table 2. Clinical symptoms and laboratory results on admission $(\mathrm{n}=19)$

\begin{tabular}{lc}
\hline Variable & n (\%) \\
\hline Symptoms (days)* & \\
Projectile vomiting & $15(78.9)$ \\
Non-bilious vomiting & $5(26.3)$ \\
Constipation/infrequent stooling & $5(26.3)$ \\
Poor weight gain & $13(68.4)$ \\
Laboratory results* & \\
Hypoalbuminemia $(<27 \mathrm{~g} / \mathrm{L})$ & $6(31.6)$ \\
Hyponatremia $(<136 \mathrm{~mm} / \mathrm{L})$ & $7(36.8)$ \\
Hypokalaemia $(<3.5 \mathrm{~mm} / \mathrm{L})$ & $6(31.6)$ \\
Hypochloraemia $(<95 \mathrm{mmol} / \mathrm{L})$ & $8(42.1)$ \\
High urea concentration $(>6.1 \mathrm{mmol} / \mathrm{L})$ & $3(15.8)$ \\
High pH $(>7.45)$ & $15(78.9)$ \\
Low PaCO ${ }_{2}(<33 \mathrm{mmHg})$ & $11(57.9)$ \\
Low bicarbonate level $(<28 \mathrm{mmol} / \mathrm{L})$ & $5(26.3)$ \\
Raised infection markers & $1(5.3)$ \\
$*$ More than one option could be present. & \\
\end{tabular}

No mention was made of visible peristaltic waves in any of the patient files. In four patients $(21.1 \%)$, the presence of a palpable olive was noted. Ten patients $(52.6 \%)$ were clinically dehydrated on admission: seven mild, two moderate and one severe.

\section{Management}

Seventeen patients (89.5\%) underwent a Ramstedtpyloromyotomy, all via a small right upper quadrant transverse incision. None of the patients was treated with minimally invasive surgery. Two patients $(10.5 \%)$ received atropine as their definitive treatment. Both these patients regained passage of gastric content within a week after treatment was initiated.
Of the 13 patients with time of the surgery recorded, seven $(53.8 \%)$ were operated on 11 to 30 hours after admission, while two patients $(15.4 \%)$ were operated on $>50$ hours after admission. The remaining four patients $(30.8 \%)$ were operated on 31 to 50 hours after admission. None of the patients was operated on in the first 10 hours after admission. The median time of surgery after admission was 30 hours.

Eight patients $(42.1 \%)$ received atropine as part of their initial management. Complications reported during and after the surgery included mucosal perforation, which was corrected during the operation $(\mathrm{n}=1)$, incomplete pyloromyotomy, which required revisions $(\mathrm{n}=2)$, systemic sepsis $(\mathrm{n}=2)$ and wound sepsis $(n=1)$. The causative organism for one of the patients with systemic sepsis was identified as coagulasenegative Staphylococcus. Other complications $(n=6)$ reported included postoperative bleeding, accidental injury of the duodenum and difficult intubation.

\section{Outcomes}

Nine patients $(75.0 \%)$ were ventilated postoperatively. In ten patients $(66.7 \%)$, feeds were initiated within 6-12 hours postoperatively, while three patients $(20.0 \%)$ received feeds after 24 hours (Table 3 ). One patient $(9.1 \%)$ vomited for 7 days postoperatively, while in six patients no mention of postoperative vomiting was found. There was no reported mortality among the patients. The length of stay ranged from 2 to 60 days (median 7 days).

Table 3. Postoperative characteristics

\begin{tabular}{lc}
\hline Variable & $\mathbf{n}(\mathbf{\%})$ \\
\hline Time until postoperative feeds (hours) $(\mathbf{n}=\mathbf{1 5})$ & \\
$6-12$ & $10(66.7)$ \\
$13-24$ & $2(13.3)$ \\
$>24$ & $3(20.0)$ \\
Postoperative vomiting (days) $(\mathbf{n}=\mathbf{1 1})$ & \\
0 & $2(18.2)$ \\
1 & $4(36.4)$ \\
2 & $3(27.3)$ \\
3 & $1(9.1)$ \\
$>3$ & $1(9.1)$ \\
Length of stay of all the patients (days) $(\mathbf{n}=\mathbf{1 9})$ & \\
$0-10$ & $12(63.2)$ \\
$11-20$ & $2(10.5)$ \\
$21-30$ & $1(5.3)$ \\
$31-40$ & $1(5.3)$ \\
$41-50$ & $2(10.5)$ \\
$51-60$ & $1(5.3)$ \\
& \\
& \\
&
\end{tabular}




\section{Discussion}

Universitas Hospital is a tertiary hospital in Bloemfontein and is the paediatric surgery referral centre for the Free State, Northern Cape, Lesotho and part of the Eastern Cape. At the time of the study, there was only one qualified paediatric surgeon employed at the hospital. The hospital serves a population of approximately 6.1 million people. This drainage area constitutes an extensive geographical area. Travel distances from referring hospitals vary from 3 to $1100 \mathrm{~km}$ and may require 10 to 12 hours driving time. Patients referred from Lesotho have the additional problem of a border crossing, which is time-consuming. The patient and parent(s) need to apply for a passport before being referred, which may take up to 14 days.

Most of the patients in this study were male (84.2\%), which is in keeping with the literature. ${ }^{2}$ However, the traditional teaching that IHPS is a disease of first-borns was not confirmed. Almost $80 \%$ of patients presented with the typical symptom of projectile vomiting. The second most common presenting symptom in our population was poor weight gain (68.4\%), pointing to the late presentation of patients.

Only four patients $(21.1 \%)$ had a palpable olive. The presence of an olive is thought to be pathognomonic of this condition, warranting no further investigations. ${ }^{9}$ The lack of experience of the examining doctor could play a role in this under-representation.

Metabolic alkalosis was present in almost $80 \%$ of the patients. Of concern was the low levels of serum albumin in a third of the patients, indicating a long delay before these patients arrived at the hospital for definitive treatment. Another reason for this may be the general incidence of premorbid malnutrition among the population served by Universitas Hospital. Two patients $(10.5 \%)$ presented with systemic sepsis. This could have been incidental or an indicator of a lengthy presentation before medical assistance was sought.

None of the patients underwent surgery within 10 hours after admission. In most cases $(84.6 \%)$, patients had their surgery 11 to 50 hours after admission, while two patients $(15.4 \%)$ only underwent surgery after 50 hours. Because of the longstanding presence of the gastric outlet obstruction, the patients in this study might have needed more time to be stabilised. This may have contributed to the long delay between admission and surgery.

Nine patients $(75.0 \%)$ needed ventilatory support postoperatively. The reason for this is unclear as it is hospital policy that alkalosis should be completely reversed before a patient is taken to theatre. This is to avoid the known complication of a lack of respiratory drive in the presence of hypocarbia. Patients with such a poor nutritional status that could compromise their ability to breathe normally would also not be selected for surgery. The necessity of ventilation postoperatively in such a large number of patients warrants further investigation.

According to the literature, feeding can resume as early as four hours following surgery. ${ }^{12}$ In our study, ten patients $(66.7 \%)$ received feeds within 12 hours and two
(13.3\%) within 24 hours post-surgery. Research shows that postoperative vomiting is self-limiting and does not affect hospital stay or increase postoperative complications. ${ }^{13}$ In our setting, a more aggressive feeding plan might decrease postoperative length of stay, which was a median of 7 days at the time of the study.

In this study, one patient (5.3\%) had a mucosal injury, which was corrected during the surgery, and two patients (10.5\%) needed revisions for incomplete pyloromyotomy. In a study ${ }^{14}$ analysing 2830 pyloromyotomies, only $0.29 \%$ of the 1028 open procedures resulted in mucosal perforation and $0.29 \%$ in incomplete pyloromyotomy. Bleeding from the pyloromyotomy edges is a well-described occurrence that is seldom a cause for concern due to its self-limitation as soon as the pylorus is placed back into the abdomen. ${ }^{15}$ The surgeon wants to steer away from cauterisation (the traditional way of preventing bleeding) to avoid mucosal injury.

Two patients $(10.5 \%)$ received atropine as their definitive treatment and in both cases the pathology was successfully resolved. The reason for this can be that it was the natural course of the disease and would have resolved in any case. Eight patients $(42.1 \%)$ received atropine as part of their initial management. These patients had prolonged symptoms and signs and severely deranged laboratory results. It was thought that the resuscitation to get them fit for surgery would take time. Atropine was given empirically in the interim during this resuscitation period to give the patients the benefit of possible resolution of the condition by medical treatment.

According to our experience, we recommend that atropine can be used as an empirical adjunct in the management of patients with IHPS presenting late and having severe diabolic derangements. Atropine can be given during the resuscitation/ stabilisation period before surgery. This management might cure the condition in the interim and save the patient possible surgery, as we have seen in two of our patients. We do not recommend atropine as the only treatment option replacing surgery because of the long period it takes to cure the condition and the long hospital stay it requires.

No mortality was reported for any of the patients, despite late presentation and some of the patients in desperate nutritional conditions. This corresponds with findings from a study at a tertiary hospital in KwaZulu-Natal, which included 63 patients. $^{7}$ It is essential that the timing of surgery and the selection of patients who should not undergo surgery be considered with caution.

\section{Limitations}

Study limitations include the small study size and the patient files with vital information either not recorded or missing, as is common in retrospective studies.

Student researchers, with limited to no clinical experience, collected the data. Information might have been overseen, as they were not familiar with the terminology of note keeping in the files. 


\section{Conclusion}

Infantile hypertrophic pyloric stenosis is a rare condition in South Africa. In this small series of 19 patients, the gender distribution and age at presentation were in keeping with the literature but not the birth order. Likewise, the classic presenting symptom of non-bilious projectile vomiting was present in an overwhelming majority. However, poor weight gain is not part of the symptomatology in developed countries, whereas in our study population almost $70 \%$ had poor weight gain. This condition is often misdiagnosed at our referring centres.

The majority of patients underwent surgery. Two patients needed medical management due to very poor general health. The outcome of these patients was favourable. There is a long delay before surgery after admission, once again emphasising the poor general health and biochemical deranged state the patients present at the hospital. In these patients, the empirical treatment with atropine on admission might be advantageous. This highlights the value of prompt referral to an appropriate paediatric surgical centre for stabilisation and surgical intervention.

\section{Recommendations}

The high postoperative ventilation rate warrants further investigation. A more aggressive attitude towards postoperative initiation of feeds should be followed, even in the presence of postoperative vomiting. Staff training and awareness of IHPS should be undertaken during outand in-reach programmes to hospitals in the referral area of Universitas Hospital.

\section{Acknowledgements}

We thank the personnel from the records department at Universitas Hospital for file retrieval, Dr SM le Grange, Head of Clinical Unit/Senior Lecturer: Paediatric Surgery, Department of Surgery, UFS, for editing the final product, and Ms T. Mulder, medical editor, Faculty of Health Sciences, UFS, for technical and editorial preparation of the manuscript.

\section{Conflict of interest}

\section{None}

\section{REFERENCES}

1. Ceccarelli M, Villirillo A, Assanta N, Balsano L, Chiaravalloti G. Hypertrophic pyloric stenosis in infants. A retrospective study of cases observed in the years 1970-1990. Pediatr Med Chir. 1992;14(4):441-3. [Article in Italian] [PMID: 1461785]
2. Burge DM, Griffiths DM, Steinbrecher HA, Wheeler RA, eds. Paediatric Surgery. 2nd ed. London: Hodder Arnold, 2005:1915.

3. Rosenthal YS, Chodick G, Grossman Z, Shalev V, Koren G. The incidence of infantile hyperthropic pyloric stenosis and its association with folic acid supplementation during pregnancy: a nested case-control study. J Pediatr Surg. 2018 [in press]. doi: 10.1016/j.jpedsurg.2018.05.005

4. Emmink B, Hadley GP, Wiersma R. Infantile hypertrophic pyloric stenosis in a Third-World environment. S Afr Med J. 1992;82(3):168-70. [PMID: 1519137]

5. Pedersen RN, Garne E, Loane M, Korsholm L, Husby S; EUROCAT Working Group. Infantile hypertrophic pyloric stenosis: a comparative study of incidence and other epidemiological characteristics in seven European regions. J Matern Fetal Neonatal Med. 2008;21(9):599-604. [PMID: 18828050] doi: 10.1080/14767050802214824

6. Tadesse A, Gadisa A. Infantile hypertrophic pyloric stenosis: A retrospective study from a tertiary hospital in Ethiopia. East Cent Afr J Surg. 2014;19(1):120-4.

7. Saula PW, Hadley GP. Hypertrophic pyloric stenosis in the third world. Trop Doct. 2011;41(4):204-10. [PMID: 21914675] doi: 10.1258/td.2011.110145

8. Macdessi J, Oates RK. Clinical diagnoses of pyloric stenosis: a declining art. BMJ.1993;306(6877):553-5. [PMID: 8461768] doi: $10.1136 /$ bmj.306.6877.553

9. White MC, Langer JC, Don S, DeBaun MR. Sensitivity and cost minimization analysis of radiology versus olive palpation for the diagnosis of hypertrophic pyloric stenosis. J Pediatr Surg. 1998:33(6);913-7. [PMID: 9660228] 10.1016/S00223468(98)90673-X

10. Turial S, Enders J, Schier F, Santos M. Comparison of a novel technique of the microlaparoscopic pyloromyotomy to circumbilical and Weber-Ramstedt approaches. J Gastrointest Surg. 2011;15(7):1136-42. [PMID: 21538191] doi: 10.1007/ s11605-011-1531-9

11. de Laffolie J, Turial S, Heckmann M, Zimmer KP, Schier F. Decline in infantile hypertrophic pyloric stenosis in Germany in 2000-2008. Paediatrics. 2012;129(4):901-6. [PMID: 22430445] doi: 10.1542/peds.2011-2845

12. Turnock RR, Rangecroft L. Comparison of postpyloromyotomy feeding regimens in infantile hypertrophic pyloric stenosis. J R Coll Surg Edinb. 1991;36(3):164-5. [PMID: 1920229]

13. van der Bilt JD, Kramer WL, van der Zee DC, Bax NM. Early feeding after laparoscopic pyloromyotomy: the pros and cons. Surg Endosc. 2004;18(5):746-8. [PMID: 15026900] doi: 10.1007/s00464-003-9076-y

14. Hall NJ, Eaton S, Seims A, et al. Risk of incomplete pyloromyotomy and mucosal perforation in open and laparoscopic pyloromyotomy. J Pediatr Surg. 2014;49(7):10836. [PMID: 24952793] doi: 10.1016/j.jpedsurg.2013.10.014

15. Mulholland MW, Doherty GM, eds. Complications in Surgery. 2nd ed. Philadelphia: Lippincott Williams \& Wilkins, 2011:733. 